\title{
Detection of Small Sea-Surface Targets with a Search Lidar
}

\author{
Johan C. van den Heuvel, Herman H.P.Th. Bekman, Frank J.M. van Putten, Leo H. Cohen \\ TNO Defence, Security and Safety, Oude Waalsdorperweg 63, The Hague, The Netherlands
}

\begin{abstract}
Naval operations in the littoral have to deal with the threat of small sea-surface targets. These targets have a low radar cross-section and low velocity, which makes them hard to detect by radar in the presence of sea clutter. Typical threats include periscopes, jet skies, FIAC's, and speedboats. Search lidars on board naval vessels can provide detection capability for small sea-surface targets. Lidar measurements at the coast have shown a very good signal-to-clutter ratio with respect to buoys located up to $10 \mathrm{~km}$ from the shore were the lidar system was situated. The lidar clutter is much smaller than the radar clutter due to the smoothness of the sea surface for optical wavelengths, thus almost all laser light is scattered away from the receiver. These results show that due to the low clutter a search lidar is feasible that can detect small sea-surface targets. The concept of a search lidar is presented and its performance is derived from system models. By using a high rep-rate laser and a variable beam divergence the search time can be limited. The design of a search lidar based on a commercially available high power and high rep-rate laser is shown.
\end{abstract}

Keywords: Laser, laser range finder, small targets, lidar, detection

\section{INTRODUCTION}

Detection of small sea-surface targets, like periscope tubes, jet skies, swimmers and small boats, is important both for civil and naval scenarios, e.g. 'man overboard', illegal immigration, drugs transport and asymmetric threats. However, the detection of these targets using current radar systems is difficult due to the small radar cross-section and low velocity of these targets and the presence of strong radar clutter for the sea surface. Stealth constructions will reduce radar reflections of targets even further.

The detection of sea-surface targets is based on the low reflection of the sea and the strong reflection of hard targets. Experiments from the Meetpost Noordwijk Netherlands (in 1996 and 2000) and along the Irish west coast during the PARFORCE trials ${ }^{1}$ in 1999 and 2000 confirmed the low sea reflection. Other experiments learned that relatively strong signals were returned from small hard targets, e.g. poles and streetlamps, a principle that is used by laser range finders. ${ }^{2}$

Lidar systems are particularly suitable as a search-around system for the detection of small (stealth) targets at the sea surface. Their small instantaneous field-of-view, small pulse durations and high power combine a high spatial resolution with a large detection range. Moreover, it is shown here that small sea-surface targets give strong reflections in comparison with the reflections from the sea surface. This results in a large signal-to-clutter-ratio. Operation during periods of radio silence is possible since detection of optical radiation with standard radio equipment and vice versa is not possible. Laser radiation can only be detected with special equipment, e.g., laser warning systems.

The maximum detection range of a search lidar depends not only on the system properties but also on the atmospheric condition. The latter plays a dominant role, especially under low visibility conditions because both the transmitted laser radiation and the backward reflected radiation is attenuated. Consequently, the maximum detection range depends on the system properties, on the target reflection properties, and on the atmospheric visibility.

Search lidars are intended for the detection of targets and measurement of their position (range and heading). Speed and heading can be obtained from multiple measurements. It is possible that search lidars can also be used for classification or identification of non-cooperating long range targets.

The principle of a search lidar consists of a scanning laser beam with a relatively small instantaneous-field-of-view (IFOV) in combination with an optical receiver, aligned with the laser beam. Scanning is done in azimuth and elevation

Laser Radar Technology and Applications XII, edited by Monte D. Turner, Gary W. Kamerman, Proc. of SPIE Vol. 6550, 65500V, (2007) · 0277-786X/07/\$18 · doi: 10.1117/12.719292 
to cover the sea-surface around the ship. The receiver measures part of the backward scattered laser radiation from targets within the IFOV of the laser beam. Range information is obtained by using pulsed lasers in combination with a receiver that records the time-of-flight of the received laser pulse. A sketch of a basic search lidar is shown in Figure 1 with diagrams of the scanning system and the interaction at the sea surface.

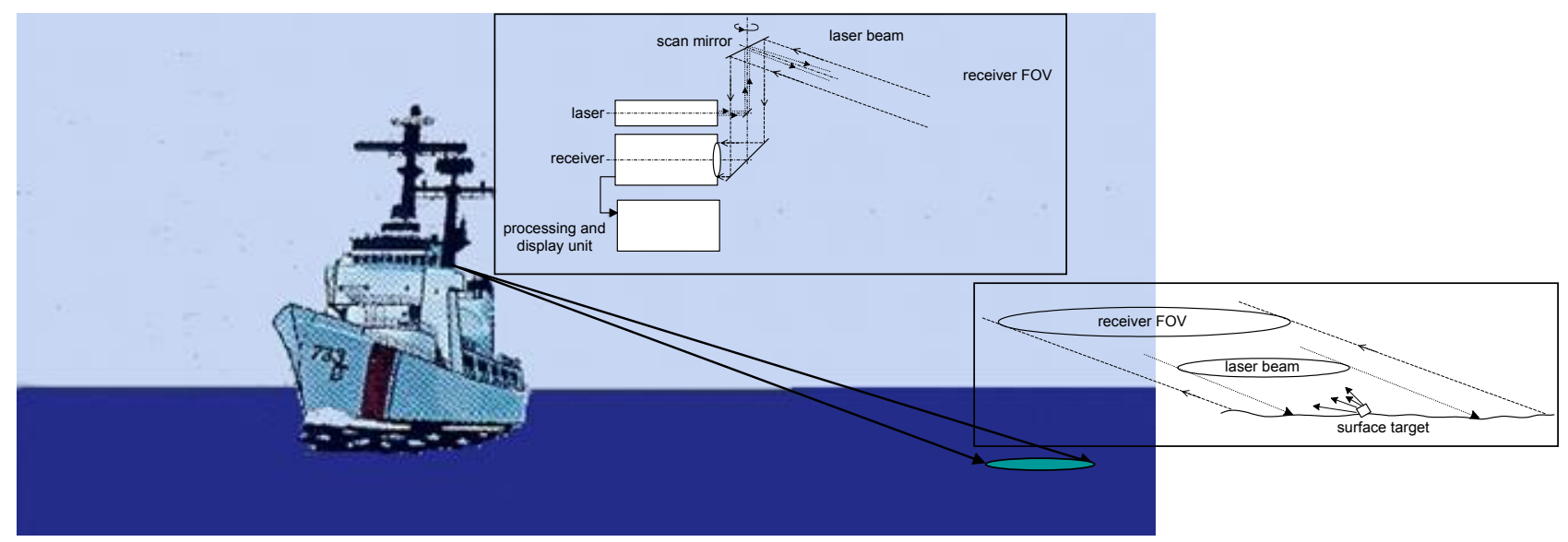

Figure 1 Sketch of a search lidar with diagrams of scanning system and sea-surface interaction.

The reflection of the sea surface is very important in this context, but theory is complex, difficult to handle and commonly used to predict reflections from the sun and the sky towards an observer but not to predict reflections of mono-static lidar systems or laser range finders. Therefore, we have measured the target and the sea reflection with our backscatter lidars at $1.06 \mu \mathrm{m}$ and $1.57 \mu \mathrm{m}$ near the Dutch coast. Within this context a lidar operating at the eye-safe wavelength of $1.57 \mu \mathrm{m}$ has been developed for eye-safety reasons. At this wavelength the radiation is strongly absorbed in the eye and badly focused on the retina. The development of this laser and the lidar receiver is briefly discussed.

\section{EXPERIMENTAL SET-UP}

To verify the search lidar concept, initial measurements were carried out on small buoys near Scheveningen harbor (North Sea coast, The Netherlands) at distances between 1 and $10 \mathrm{~km}$ with the $1.06 \mu \mathrm{m}$ system at a pulse energy of $20 \mathrm{~mJ}$ at $20 \mathrm{~Hz}$.

Although the lidar operating at $1.06 \mu \mathrm{m}$ is an excellent tool for a feasibility study, it is not eye-safe. Therefore, an eyesafe lidar has been developed operating at a wavelength of $1.57 \mu \mathrm{m}$, which is absorbed in the eye and not focused on the retina. It is possible that the reflection properties of surfaces and the scattering properties of atmospheric particles at the eye-safe wavelength will be different from the $1.06 \mu \mathrm{m}$ wavelength. However, different references indicate that the differences are marginal. ${ }^{3,4,5,6}$ Molecular transmission of the atmosphere calculated with Modtran showed that under normal atmospheric conditions the transmission at $1.06 \mu \mathrm{m}$ and $1.57 \mu \mathrm{m}$ is comparable. Therefore, no special molecular effects are expected. The relatively strong absorption lines at $1.1 \mu \mathrm{m}$ and $1.4 \mu \mathrm{m}$ are caused by water vapor but are outside the applied laser wavelengths.

The lidar system at $1.57 \mu \mathrm{m}$ is depicted in Figure 2. The green box contains the OPO laser wavelength converter, which converts the pump laser wavelength of $1.06 \mu \mathrm{m}$ into the more eye-safe wavelength of $1.57 \mu \mathrm{m} .^{7,8,9}$ The white box on top of the green box contains the receiver telescope and the detector. The white box right next to the green box is the 1.06 $\mu \mathrm{m}$ pump laser.

The pump laser for the OPO is a Q-switched Nd:YAG laser. The maximum output is $350 \mathrm{~mJ}$ per pulse with a pulse length of $5 \mathrm{~ns}$ at a rep-rate of $20 \mathrm{~Hz}$. In the OPO the laser radiation at $1.06 \mu \mathrm{m}$ is converted to $1.57 \mu \mathrm{m}$. The conversion takes place in a non-linear crystal. To increase the non-linear conversion efficiency, it is beneficial to increase the laser 
power density by focusing the laser beam into the crystal. However there is a limit on the maximum power density that the crystal can withstand.

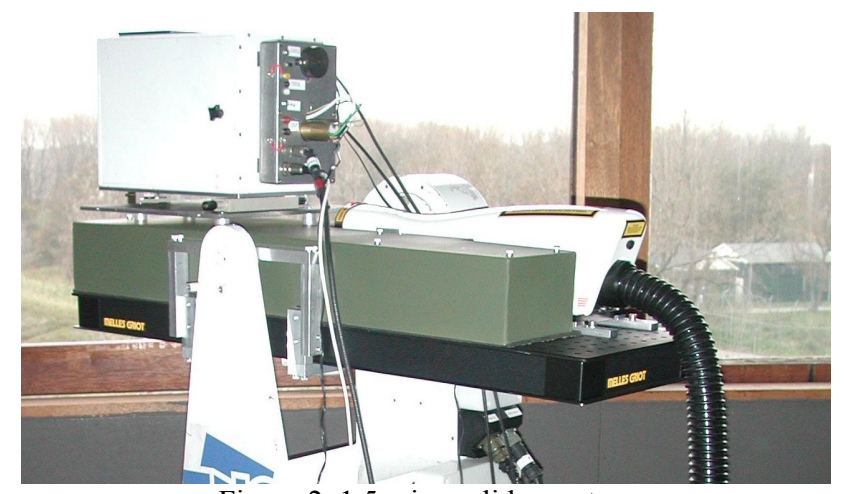

Figure 21.5 micron lidar system

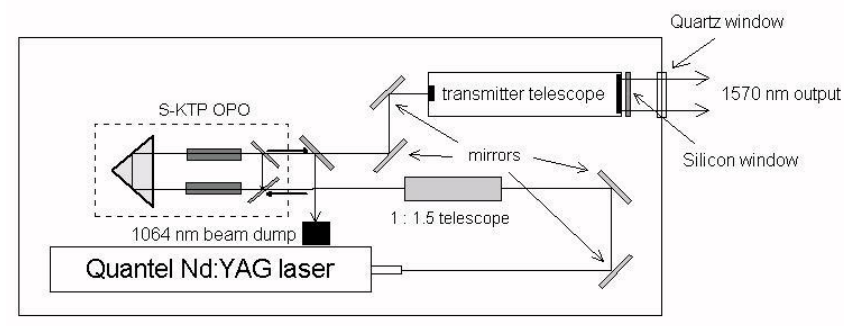

Figure 3 Optical layout of eye safe lidar transmitter.

We have made an OPO design that maximizes the non-linear conversion efficiency by using two crystals instead of one, and by using S-KTP instead of KTP crystals which has a higher damage threshold. We ended up with a ring cavity design. The OPO laser is connected to an optical telescope and in addition some filters are placed in the beam line to completely block the eye-hazardous laser pump light. The layout of the transmit section of the LIDAR is shown in Figure 3. The LIDAR system was operated with maximum pump pulse energy of $205 \mathrm{~mJ}$ at a rep-rate of $10 \mathrm{~Hz}$. The output from the transmitter at $1570 \mathrm{~nm}$ was around $30 \mathrm{~mJ}$. The receiver is constructed using a $750 \mathrm{~mm}$ telescope, with an optical aperture of $125 \mathrm{~mm}$ diameter. An InGaAs APD detector with a diameter of $80 \mu \mathrm{m}$ was used.

\section{EXPERIMENTAL RESULTS}

A suitable location with small sea-surface targets was searched along the Dutch coast, to investigate the principle of detecting small targets at the sea surface. The location of interest should have some small objects in the water, preferably at least several kilometers from the observation point. A good location appeared to be the southern Scheveningen harbor pier with a length of about $600 \mathrm{~m}$ at approximately $\left(52^{\circ} 6^{\prime} \mathrm{N} ; 4^{\circ} 15^{\prime} \mathrm{E}\right)$. From this position several buoys at distances between $1 \mathrm{~km}$ and $10 \mathrm{~km}$ are visible.

A magnified portion of Hydrographic Map 1801.7 is shown on the left of Figure 4. The map (courtesy Royal Netherlands Navy, 'Dienst der Hydrografie') shows the buoys near the Scheveningen harbour entrance. The rectangles in the background of the map are approximately $1 \times 2 \mathrm{~km}^{2}$. The lidar was positioned at the end of the southern pier, near the green traffic light (near text 'Obtns'). Shown on the right side of Figure 4 is a view over the sea in NW direction from the southern harbour pier, with the lidar in the front and the buoys in the back. Red circles mark the four buoys of interest that are from left to right: DR-B, Drain-W, Dr-A, and Drain-E. The ranges of the buoys are DR-B at $1.4 \mathrm{~km}$, Drain-W at $1.9 \mathrm{~km}$, Dr-A at $1.2 \mathrm{~km}$, and Drain-E at $1.6 \mathrm{~km}$. 

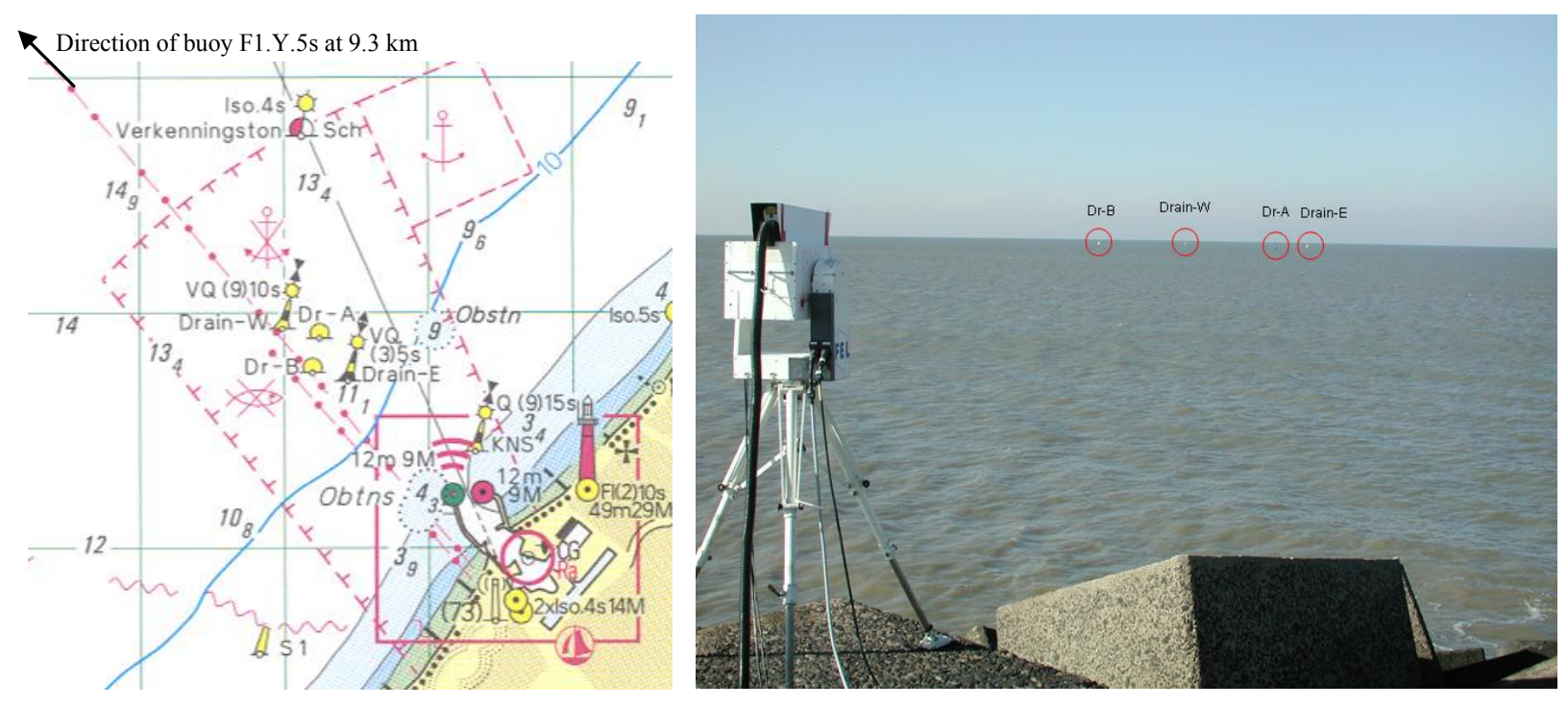

Figure 4 Magnified portion of Hydrographic Map 1801.7 (left) and the lidar situated at the end of the southern pier (right).

The lidar at $1.06 \mu \mathrm{m}$ was set up at the end of the southern Scheveningen harbor pier at about $500 \mathrm{~m}$ from the surf zone and the measurements were carried out in north-westerly directions towards open sea. No shipping traffic was observed during the experiments. The weather conditions were good. Cloudless sky, wind speed 6-7 m/s, wind direction 70 degrees, air temperature $12^{\circ} \mathrm{C}$ and air pressure $1031 \mathrm{hPa}$. The water temperature was not specified in the weather report, but based on previous information in this time of the year it is assumed that the water temperature is about $16{ }^{\circ} \mathrm{C}$. The wave height was about $0.5 \mathrm{~m}$ and whitecaps were only occasionally observed.

Horizontal lidar measurements were carried out at 10 different elevation angles from $+0.46^{\circ}$ degrees down to $-0.49^{\circ}$ to observe buoy reflections at different heights. Results obtained from one of these measurements under the elevation angle of $-0.24^{\circ}$ are presented in Figure 5. The lidar is in the right most position in this figure. Reflections from the buoys are indicated with red circles. The upper right yellow circle is not a buoy but probably the reflection from a sea gull. Distances between the concentric range indicators are $500 \mathrm{~m}$. The regular fringes at ranges larger than $2 \mathrm{~km}$ (in the most left part in the figure) are reflections from the wave tops.

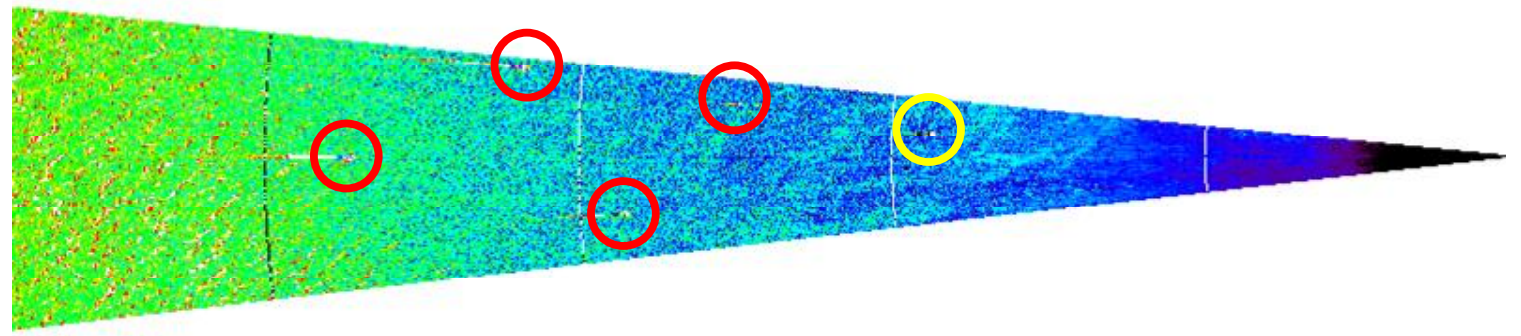

Figure 5 Results of a horizontal lidar scan over an azimuth angle interval of 12 degrees and at an elevation angle of $-0.24^{\circ}$.

Actually, the data-density in Figure 5 is too high to emphasize the reflections from the small surface targets. Therefore, the data are first plotted at a higher spatial resolution to present more detail in the data. Subsequently the data is plotted using a different color scale. For example, the left panel of Figure 6 shows the same data as presented in Figure 5 but projected over a grid of $100 \times 100 \mathrm{~m}$. The right panel in Figure 6 shows the data at a somewhat lower elevation angle, recorded 13 seconds later at an elevation angle of $-0.31^{\circ}$; note that the "sea gull" reflection has moved. The pattern in the middle of this figure is caused by reflections from the waves at the sea surface. 

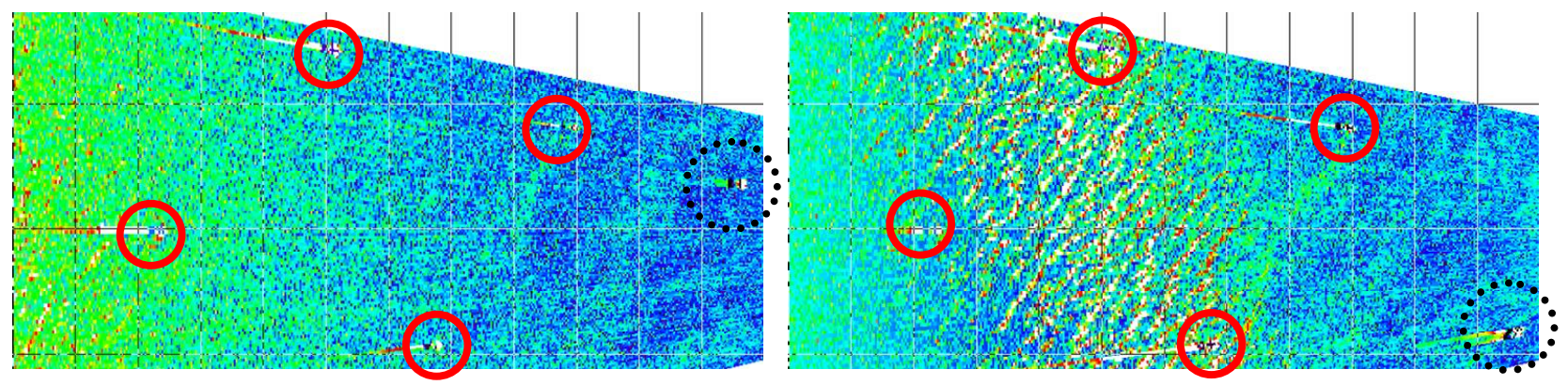

Figure 6 Two horizontal lidar scans: data of Figure 5 (left) and data at lower elevation angle 13 sec later (right).

The results in Figure 6 show that the lengths of the target reflections (duration) increase with distance, thus reflections from buoys at larger ranges have a stronger overload effect on the receiver than reflections from nearby buoys. Probably only the top fraction of nearby buoys is illuminated. For larger ranges, the beam is closer to the surface and has a wider cross-section, which results in a better illumination of the buoys, and thus in a stronger reflection. Note that the length of the reflection is more than $100 \mathrm{~m}$, which is caused by saturation of the receiver. Without saturation the duration of the return should be on the order of the laser pulse width, thus no more than $6 \mathrm{~m}$. In the right panel the surface wave reflections are at shorter ranges than those in the left panel due to the lower elevation angle.

So far, the data has been processed to visualize as much information as possible, thus over a wide dynamic range of signal amplitudes. As a result of this choice also the reflections of the atmosphere and the waves are clearly visible and the reflections from the buoys seemed to be embedded in the clutter. However, the signals from the buoys are several orders of magnitude stronger than the background thus by a proper selection of the color interval only the returns from the buoys are shown. Of course, this will depends on the range $(R)$ because the strength of the atmospheric return decreases with at least $R^{2}$ whereas the return from point targets decrease with $R^{4}$. Figure 7 shows the same data as presented in the right panel of Figure 6, but using an amplitude interval that is two orders of magnitude less sensitive. With this intensity scale both the atmospheric return and the reflection from the surface waves are no longer visible. Only the buoy reflections and the "sea gull" remain visible.

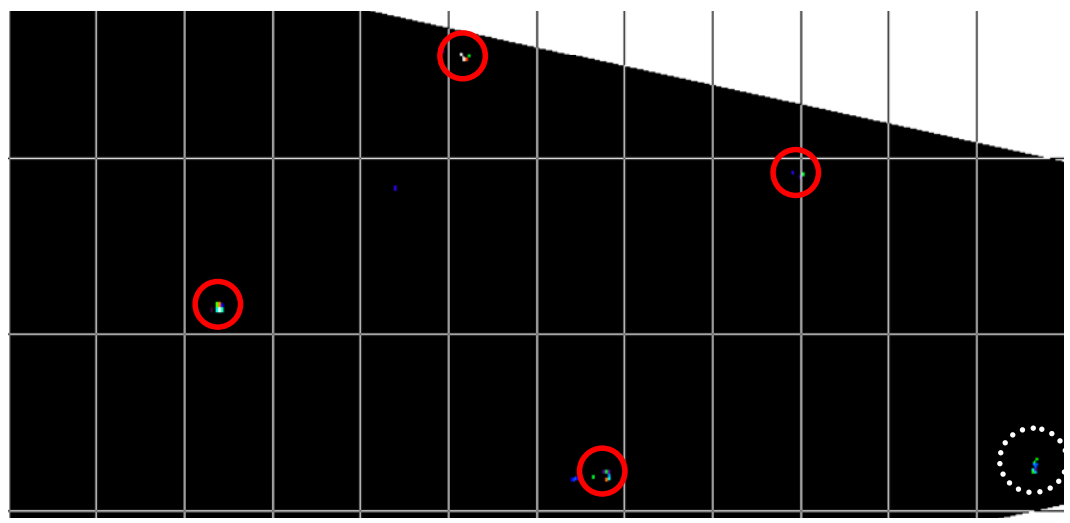

Figure 7 Same data as presented in the right panel of Figure 6 but coded in a color scale that is a factor 100 less sensitive.

During later experiments, the lidar could successfully measure the position and reflection of the buoy F1.Y.5s in northwesterly direction at a distance of $9.3 \mathrm{~km}$. The latter could not be observed with the naked eye under these prevailing conditions and only slightly using a binocular. Using the lidar, the buoy could be detected with a signal-tonoise ratio of about 140. A screen snapshot of the waveform recorder is shown in Figure 8. The lidar waveform presented in Figure 8 was recorded with a time base of $10 \mu \mathrm{s} / \mathrm{div}$ and a pretrigger of $10 \mu \mathrm{s}$. The gradual decrease in the signal between about $10 \mu \mathrm{s}$ and $60 \mu \mathrm{s}$ represents the atmospheric backscatter, attenuated by the geometric effect and the atmospheric transmission losses. Due to the application of an analog logarithmic amplifier, which suppresses large signal amplitudes and enhances low signal amplitudes, the large dynamic range of the signal could be covered within a single 
waveform. The relatively strong peak, visible at $62 \mu$ s after the pretrigger, comes from the buoy at $9.3 \mathrm{~km}$ from the lidar. Analysis of this signal shows that the signal-to-noise ratio of this peak is a factor 140.

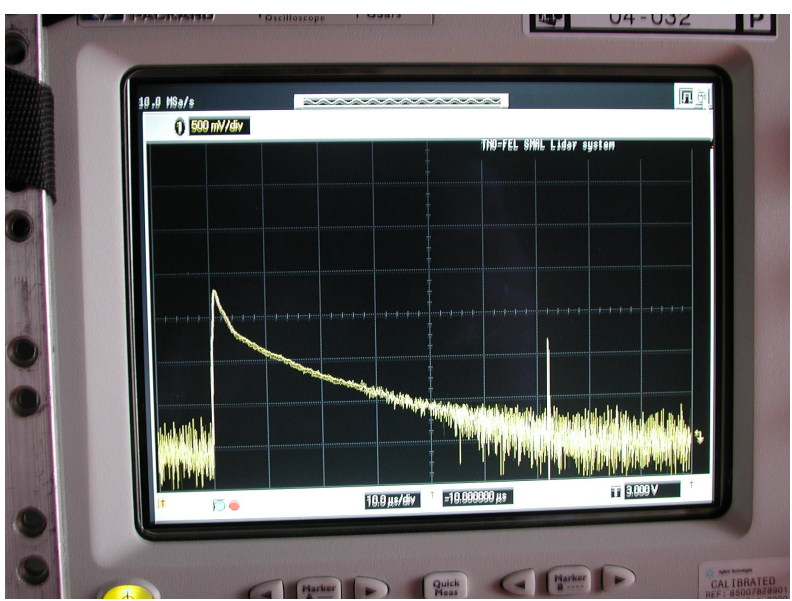

Figure 8 Photo of the oscilloscope screen with a lidar waveform showing the reflected signal from buoy Fl.Y.5s at $9.3 \mathrm{~km}$.

The $1.5 \mu \mathrm{m}$ lidar that was presented in the previous section was used at the same location as the $1.06 \mu \mathrm{m}$ (but a year later) to check whether the same favorable results could be achieved at this eye-safe wavelength. Since this system does not have a logarithmic receiver for the atmospheric return signal, we can only show the linear plot of the return of the buoys. Figure 9 shows the lidar return signals for the four buoys, which clearly show a good signal-to-noise ratio.

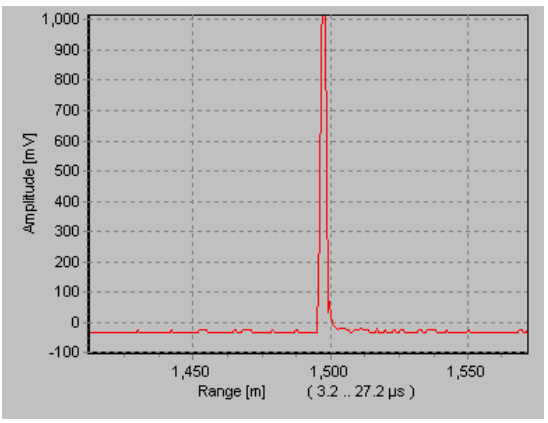

Dr-B

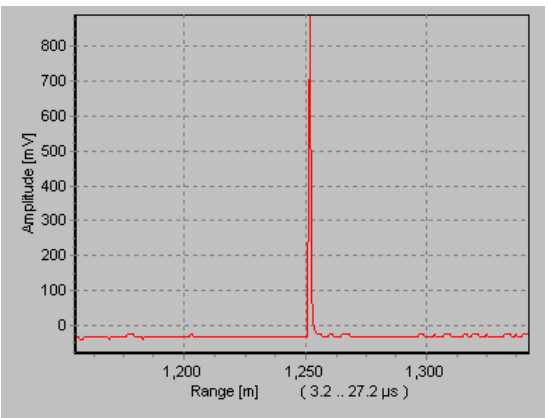

Dr-A

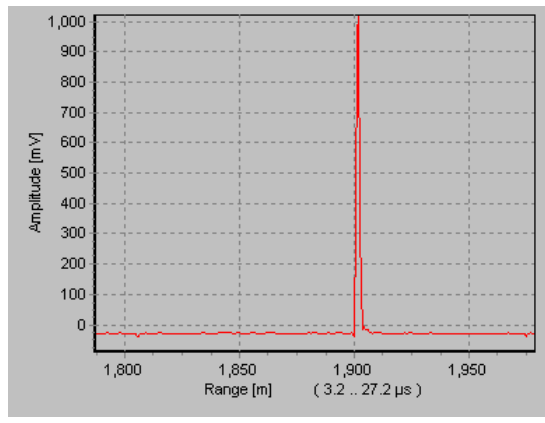

Drain-W

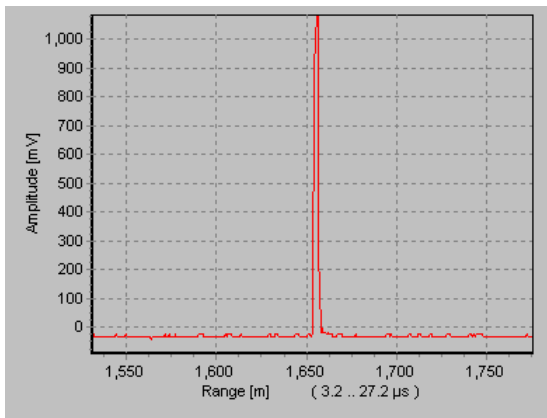

Drain-E

Figure 9 Laser return signals of the buoys measured with the $1.5 \mu$ m lidar.

Since the lidar is eye-safe, we can also aim the system without risk at manned platforms. Figure 10 shows that is it feasible to detect a small sailing boat at $3.4 \mathrm{~km}$. Given the good signal-to-noise ratio, much larger ranges are possible. 

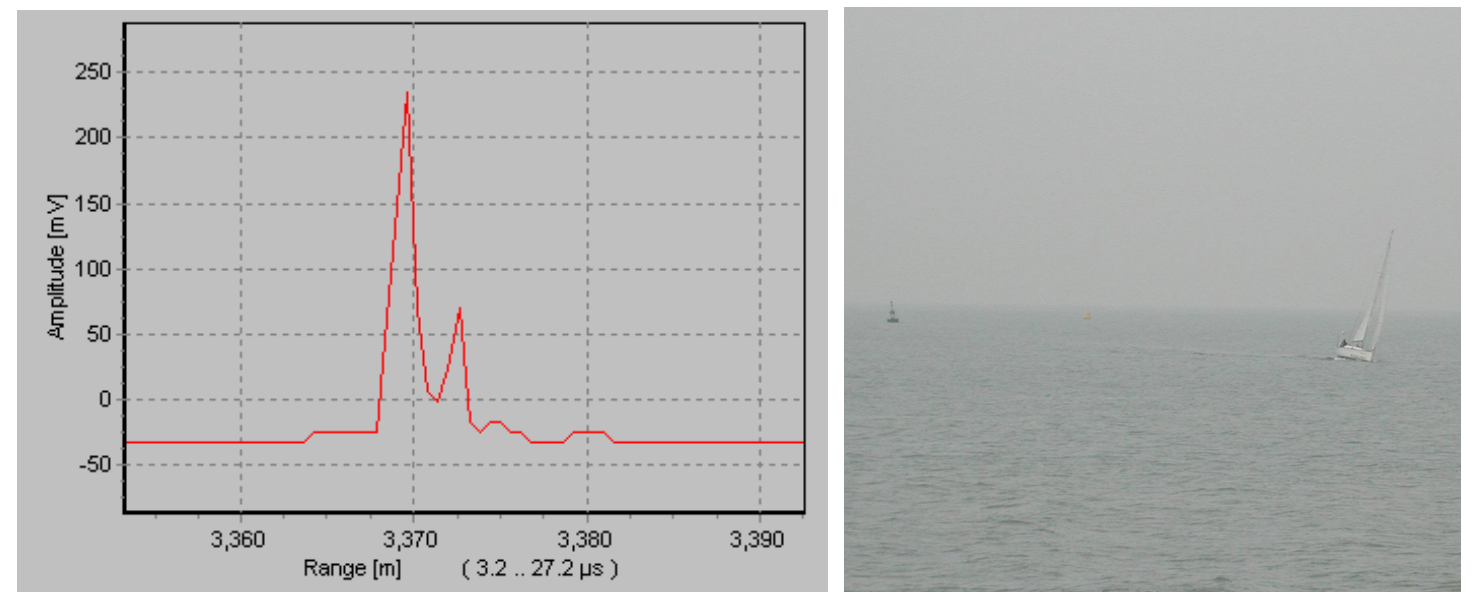

Figure 10 Laser range profile of a small sailing boat at $3.4 \mathrm{~km}$. The photograph was taken at short range.

\section{PERFORMANCE CALCULATIONS}

The performance of search lidars can be modeled by taking into account the system parameters, the target properties and the transmission of the atmosphere. These parameters can be used in the laser range finder equation with the assumption that the cross section of the laser beam is larger than the cross section of the target. The detected (received) laser power is given by

$$
P_{D}=\frac{\eta A_{T}}{R^{4}} \frac{4 P_{L} A_{D}}{\pi^{2} \psi^{2}} T_{O} T_{A}^{2}+N,
$$

where $A_{D}$ and $A_{T}$ are the receiver and the target area; $N$ is the noise power; $P_{D}$ and $P_{L}$ are the received (detector) and the laser power; $R$ is the range; $T_{O}$ and $T_{A}$ are the transmission of optics and atmosphere; $\eta$ is the target's reflection coefficient; and $\psi$ is the laser beam divergence.

Targets may be identified when the returned signal is stronger than a selected minimum level or threshold, which is a function of an acceptable average false alarm rate and the receiver noise level. In turn, the probability of detection can be calculated from the strength of the returned target signal, the noise level and the selected threshold. ${ }^{10,11,12}$ The threshold pulse energy is calculated with the following formula:

$$
E_{\text {thr }}=N E P \sqrt{B W} S N R T_{\text {pulse }} \text {, }
$$

where $B W$ is the bandwidth of the receiver, $S N R$ is the signal-to-noise ratio, and $T_{p u l s e}$ is the pulse duration. For a false alarm rate of $10^{-3}$ and a probability of detection of $95 \%$, the required $S N R$ is 7 .

Atmospheric transmission can be estimated from the visibility and applying the proposed wavelength correction. ${ }^{13}$ The atmospheric transmission $T_{A}$ is approximated by the following empirical equation:

$$
T_{A}=\exp (-\mu R) \text { with } \mu=\frac{3.912}{V}\left(\frac{\lambda_{r}}{\lambda}\right)^{q} \text { and } q=0.585 \mathrm{~V}^{1 / 3},
$$

where $R$ is the range, $V$ is the visibility, and $\lambda$ is the laser wavelength. The reference wavelength $\lambda_{r}$ is $0.55 \mu \mathrm{m}$.

Generic targets for a littoral scenario were obtained in a separate study. These targets are listed in Table 1. The minimum required laser pulse energy for the detection of these targets at a range indicated in the scenario is calculated using the following specifications of the lidar: laser beam divergence of $1 \mathrm{mrad}$, receiver diameter of $10 \mathrm{~cm}$, detector noise of $2 \times 10^{-13} \mathrm{~W} / \sqrt{\mathrm{Hz}}$, signal bandwidth of $30 \mathrm{MHz}$ and a system transmission $T_{O}$ of $50 \%$. Signal processing is assumed to be based on a false alarm rate of $10^{-3}$ and a detection probability of $95 \%$. Laser pulses of $10 \mathrm{~ns}$ will be considered. It will be assumed that the equivalent diffuse reflection coefficient of the targets is $5 \%$. Atmospheric transmission will be considered based on the visibility condition of $30 \mathrm{~km}$. Speckle effects and atmospheric turbulence are not taken into account. A list of targets and required laser pulse energy to detect these targets at the indicated ranges are summarized in Table 1. 
Table 1 Target properties and minimum laser pulse energy to detect the indicated targets in the given aspect, front view (FV) and side view $(\mathrm{SV})$, at the given range in kilometers.

\begin{tabular}{|c|c|c|c|c|c|c|c|c|c|}
\hline \multicolumn{8}{|c|}{ Target properties } & \multirow{2}{*}{\multicolumn{2}{|c|}{$\begin{array}{c}\text { Minimum laser pulse } \\
\text { energy in Joule }\end{array}$}} \\
\hline & & \multicolumn{3}{|c|}{$\begin{array}{l}\text { Dimension } \\
\quad \text { in } m\end{array}$} & \multicolumn{2}{|c|}{$\begin{array}{l}\text { Cross section } \\
\text { in } m^{2}\end{array}$} & \multirow[t]{2}{*}{$\begin{array}{c}\text { Range } \\
\text { in km }\end{array}$} & & \\
\hline$n r$ & Description & $L$ & $W$ & $H$ & $F V$ & $S V$ & & $F V$ & $S V$ \\
\hline 1 & Fishing boat & 20 & 5 & 4 & 20 & 80 & 18 & 0.39 & 0.10 \\
\hline 2 & Speed boat & 5 & 2 & 1 & 2 & 5 & 18 & 3.94 & 1.58 \\
\hline 3 & Power boat & 10 & 3 & 2 & 6 & 20 & 18 & 1.31 & 0.39 \\
\hline 4 & FIAC 2B & 14 & 4.5 & 3 & 13.5 & 42 & 18 & 0.58 & 0.19 \\
\hline 5 & FIAC 3 & 22 & 5.5 & 3 & 16.5 & 66 & 18 & 0.48 & 0.12 \\
\hline 6 & FIAC 2A & 13 & 2.7 & 2 & 5.4 & 26 & 3.7 & $5.5 \times 10^{-5}$ & $2.9 \times 10^{-5}$ \\
\hline 7 & FIAC 1B & 7 & 2.3 & 2 & 4.6 & 14 & 3.7 & $6.4 \times 10^{-5}$ & $2.9 \times 10^{-5}$ \\
\hline 8 & Jet ski & 3.1 & 1.2 & 1 & 1.2 & 3.1 & 3.7 & $25 \times 10^{-5}$ & $9.5 \times 10^{-5}$ \\
\hline 9 & RIB & 3.5 & 2 & 0.5 & 1 & 1.75 & 3.7 & $30 \times 10^{-5}$ & $17 \times 10^{-5}$ \\
\hline 10 & Patrol boat & 47 & 7.1 & 8 & 56.8 & 376 & 18 & 0.14 & 0.03 \\
\hline
\end{tabular}

The results in Table 1 show that the targets 6-9 can be detected under clear conditions with relatively low laser pulse energies, on the order of $0.3 \mathrm{~mJ}$ or less. Targets $1,4,5$ and 10 can be detected using medium laser pulse energies of less than 1 Joule. Higher pulse energies of more than 1 Joule are required to detect the targets 2 and 3.

Another point of view it is to look at the maximum detection range of the specified targets with a given search lidar. For this purpose, the same search lidar is assumed as specified earlier but now with a fixed pulse energy of $10 \mathrm{~mJ}$. The results of these calculations are presented in Table 2.

Table 2 Target properties and maximum detection ranges of the specified targets in front view (FV) and side view (SV) using a laser with a pulse energy of $10 \mathrm{~mJ}$.

\begin{tabular}{|c|c|c|c|c|c|c|c|c|}
\hline \multicolumn{7}{|c|}{ Target properties } & \multirow{2}{*}{\multicolumn{2}{|c|}{$\begin{array}{c}\begin{array}{c}\text { Maximum detection } \\
\text { range in } \mathbf{k m}\end{array} \\
\text { Visibility } 30 \mathrm{~km}\end{array}$}} \\
\hline & & \multicolumn{3}{|c|}{$\begin{array}{l}\text { Dimension } \\
\quad \text { in } m\end{array}$} & \multicolumn{2}{|c|}{$\begin{array}{c}\text { Cross section in } \\
\mathrm{m}^{2}\end{array}$} & & \\
\hline$n r$ & Description & $L$ & $W$ & $H$ & $F V$ & $S V$ & $F V$ & $S V$ \\
\hline 1 & $\begin{array}{l}\text { Fishing } \\
\text { boat }\end{array}$ & 20 & 5 & 4 & 20 & 80 & 11.2 & 13.6 \\
\hline 2 & Speed boat & 5 & 2 & 1 & 2 & 5 & 7.8 & 9.1 \\
\hline 3 & Power boat & 10 & 3 & 2 & 6 & 20 & 9.3 & 11.2 \\
\hline 4 & FIAC 2B & 14 & 4.5 & 3 & 13.5 & 42 & 10.6 & 12.5 \\
\hline 5 & FIAC 3 & 22 & 5.5 & 3 & 16.5 & 66 & 10.9 & 13.2 \\
\hline 6 & FIAC 2A & 13 & 2.7 & 2 & 5.4 & 26 & 9.2 & 11.6 \\
\hline 7 & FIAC 1B & 7 & 2.3 & 2 & 4.6 & 14 & 8.9 & 10.6 \\
\hline 8 & Jet ski & 3.1 & 1.2 & 1 & 1.2 & 3.1 & 7.2 & 8.4 \\
\hline 9 & RHIB & 3.5 & 2 & 0.5 & 1 & 1.75 & 6.9 & 7.6 \\
\hline 10 & Patrol boat & 47 & 7.1 & 8 & 56.8 & 376 & 13.0 & 15.0 \\
\hline
\end{tabular}

\section{SEARCH LIDAR DEMONSTRATOR}

Currently, we are developing a search lidar demonstrator that has a high rep-rate with moderate pulse energy in order to demonstrate the feasibility of the concept. A crucial requirement is that the search time is small enough to detect seasurface targets in time. Since the search space is limited (namely at small elevation angles below the horizon) and surface targets move relatively slow, this required search time is long enough for a search lidar to be feasible. Remember that the search lidar is in particular meant for slow moving surface targets that are difficult to detect for radar. 
The search time is proportional to the number of laser pulses required for monitoring the sea surface around the search lidar position. For a horizontal scan (or azimuth scan) over angle $\theta$, the number of pulses is

$$
N_{A}=\frac{\theta}{\psi},
$$

where $\psi$ is the laser beam divergence (taken as the instantaneous field-of-view). For a full azimuth scan of $2 \pi$ radians and a beam divergence of $1 \mathrm{mrad}$, we find that at least 6300 laser pulses are required. If the search lidar is mounted at a height $h$, then the geometrical horizon is a function of the radius of the earth $\mathrm{R}_{\mathrm{E}}$ :

$$
R_{G H}=\sqrt{2 R_{E} h} .
$$

Since Table 2 showed that the search lidar has a maximum range of around $10 \mathrm{~km}$, the minimum mounting height for full range is $10 \mathrm{~m}$, which gives a range of $11 \mathrm{~km}$ using equation (5) and $R_{E}$ is $6400 \mathrm{~km}$. The elevation scan from minimum range $R_{\min }$ to the horizon needs the following number of laser pulses:

$$
N_{E}=\frac{h}{R_{\min } \psi} .
$$

A minimum range of $150 \mathrm{~m}$ means a depression angle of $67 \mathrm{mrad}$ leading to 67 laser pulses for the vertical scan. Combining the horizontal and the vertical scan, we find a total number of 420.000 laser pulses.

The search time based on 420.000 laser pulses is $42 \mathrm{~s}$ for a $10 \mathrm{kHz}$ laser. With a pulse energy of $10 \mathrm{~mJ}$, this would amount to a laser power of $100 \mathrm{~W}$, which is certainly feasible. Note that the $10 \mathrm{kHz}$ laser gives a range-ambiguity of $15 \mathrm{~km}$, which is long enough compared to the maximum range of $11 \mathrm{~km}$. The search time of $42 \mathrm{~s}$ is sufficient for slow surface targets.

There are options to reduce the search time. For instance, an increased beam divergence of 2 mrad will reduce to search time by a factor of four. However, in order to have the same detection range, the pulse energy has to increase by a factor four as well (for the same energy density at the target). Since we only need to have the high pulse energy for maximum range, we could vary the pulse energy. However, lowering the laser pulse energy for short-range operation is less attractive from a technical point of view for the laser. Alternatively, the power-density at the surface can also be lowered by increasing the divergence of the lidar. This has the advantage that a larger area can be monitored per pulse, which in turn, decreases the system's search time. The geometries of the surface-coverage for a fixed divergence system and a variable divergence system are sketched in the left and right panels of Figure 11. The concentric circles refer to range intervals for lidar operation at different elevation angles. The ellipses refer to single-shot surface coverage at different elevation and azimuth angles of the lidar. Note that the beam divergence is enlarged for clarity. The left side shows a system with a fixed instantaneous-field-of-view (IFOV) using an equal number of pulses for short and long ranges. The right sight shows geometries of illuminated areas for a system with an IFOV that decreases with range to control the laser energy density at the target. This approach requires fewer pulses for short ranges than for larger ranges.

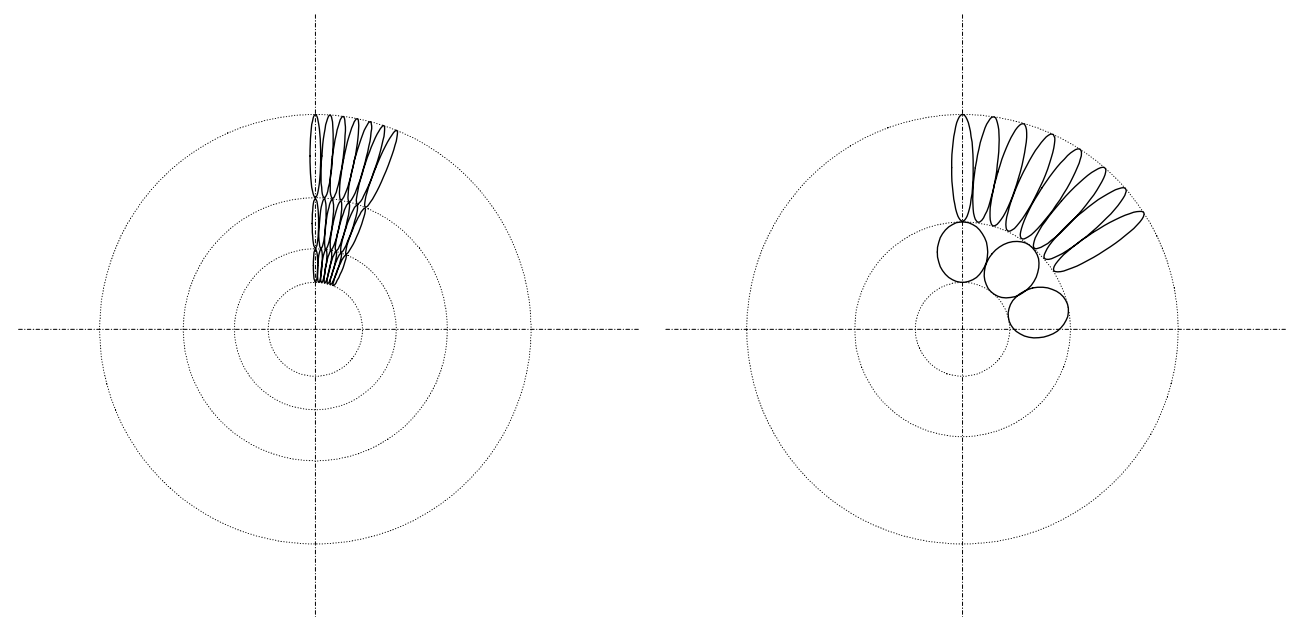

Figure 11 Surface-coverage of two search lidar concepts: one with a fixed IFOV (left) and one with a variable IFOV (right). 
For our demonstrator, we have a laser with the following specifications:

- Wavelength $1516 \mathrm{~nm}$

- Pulse repetition rate up to $3 \mathrm{kHz}$

- Pulse energy $2 \mathrm{~mJ}$ at $1 \mathrm{kHz}$

- $\quad$ Pulse width $15 \mathrm{~ns}$ at $1 \mathrm{kHz}$

- $\quad$ Beam quality $\mathrm{TEM}_{00} ; \mathrm{M}^{2}<1.2$

Since the rep-rate is lower than in the example shown above, we will demonstrate the system in a smaller azimuth and elevation scan in order to keep the search time within one minute. The pulse energy is lower than the $10 \mathrm{~mJ}$ that was used in the calculations thus the maximum range might be lower than $10 \mathrm{~km}$. For the beam steering, a commercial pan-and-tilt unit will be used that has a scan rate of $1 \mathrm{kHz} \times 1 \mathrm{mrad}$, which amounts to $1 \mathrm{rad} / \mathrm{s}$.

\section{SUMMARY AND CONCLUSIONS}

We have presented a search lidar concept for detection of small sea-surface targets. Lidar experiments at the coast have shown very low (or no) reflections from the sea surface whereas strong reflections of small targets were measured up to ten kilometers. A combination of these two phenomena have led to the conclusion that it is possible to detect small targets on the sea surface, like buoys, periscopes, jet skies, swimmers and speed boats.

A design of a search lidar demonstrator has been presented. The laser system that detects small targets at the sea surface at a range up to $10 \mathrm{~km}$ at good visibility is feasible with current laser technology. The receiver technology that was used in the performance calculations is available state-of-the-art technology. The search lidar demonstrator is currently being built.

\section{REFERENCES}

1. G.J. Kunz, G. de Leeuw, E. Becker and C.D. O'Dowd, "Lidar observations of atmospheric boundary layer structure and sea spray aerosol plumes generation and transport at Mace Head, Ireland (PARFORCE experiment)," Journal of Geophysical Research 107(D19), 11.1-11.14 (2002).

2. Kunz, G.J., et al., "Detection of small targets in a marine environment using laser radar", Proc. SPIE Vol. 5885 (2005).

3. G.H. Suits, Natural sources, Chapter 3 in W.L.Wolfe and G.J. Zissis, Eds., The infrared handbook 1978, 1978.

4. T.M. Lillesand and R.P. Kiefer, Remote sensing and image interpretation, New York: John Wiley \& Sons, 1973.

5. L.C. Rowan, A.F.H. Goetz and R.P. Ashley, "Discrimination of hydro thermally altered and unaltered rocks in visible and near infrared multispectral images," Geophysics 42(3), 522-535, 1977.

6. A.B. Kahle, Measuring spectra of arid lands, in Deserts and arid lands, El-Baz. F, Eds., pp. 195-217, Martinus Nijhoff Publishers, The Hague, The Netherlands, 1984.

7. R.L. Burnham, J.L. Kasinski, L.R. Marshall, Eye-safe laser systems, US Patent No. 5181211, 1993.

8. G.A. Rines, D.G. Rines, P.F. Moulton, "Efficient, High-Energy, KTP Optical Parametric Oscillators Pumped with 1 $\mu \mathrm{m}$ Nd-Lasers", OSA Proceedings on Advanced Solid State Lasers, Washington DC, Vol. 20, pp. 461-463, 1994.

9. B. Boulanger, M.M. Fejer, "Study of gray-tracking at 1064, 532, and 355 nm", Applied Physics Letters 65 (19) 1994.

10. R.W. Byren, "Laser Rangefinders", in The Infrared \& Electro-Optical Systems Handbook Volume 6, Chapter 2, pp. 77-116, 2nd edition, 1996.

11. G.W. Kamerman, "Laser Radar", in The Infrared \& Electro-Optical Systems Handbook Volume 6, Chapter 1, pp. 176, 2nd edition, 1996.

12. R.E. Simon, RCA Electro-Optics Handbook, Technical Series EOH-11, RCA Electronic Components, Lancaster, PA, USA, 1974.

13. F. Löhle, “Über die Lichtstreuung im Nebel”, Physik. Zeitschr. XLV, 199-204, 1944. 